\title{
Experimental evaluation of analog eigenmode transmission for short-range MIMO: array parameter independency and wideband characteristics
}

\author{
Kentaro Murata $^{1 \text { a) }}$, Naoki Honma ${ }^{2}$, Masakuni Tsunezawa ${ }^{2}$, \\ Kentaro Nishimori ${ }^{3}$, and Hisashi Morishita ${ }^{1}$ \\ ${ }^{1}$ Graduate School of Science and Engineering, National Defense Academy, \\ 1-10-20 Hashirimizu, Yokosuka, Kanagawa 239-8686, Japan \\ ${ }^{2}$ Faculty of Engineering, Iwate University, \\ 4-3-5 Ueda, Morioka, Iwate 020-8551, Japan \\ ${ }^{3}$ Faculty of Engineering, Niigata University, \\ 42-8050 Ikarashi, Niigata, Niigata 950-2181, Japan \\ a)ed14002@nda.ac.jp
}

\begin{abstract}
We proposed an analog eigenmode transmission technique, that realizes multi-stream parallel transmission in a short-range multipleinput multiple-output (SR-MIMO) communication using only 180-degree hybrid couplers. Provided the MIMO array is symmetric, this technique can orthogonalize MIMO channels independently of antenna spacing and Tx-Rx distance. Furthermore, due to wideband characteristics of a hybrid, the channel orthogonalization effect can hold over a broad band of frequencies. In this letter, its array parameter independency and wideband characteristics are quantitatively evaluated in term of channel capacity. It has been experimentally clarified that this technique achieves approximately $90-\%$ performance of ideal eigenbeam space division multiplexing (E-SDM) by digitalbeamforming despite the deviations of the above factors.
\end{abstract}

Keywords: short-range MIMO, eigenmode transmission, passive analog circuit

Classification: Antennas and Propagation

\section{References}

[1] N. Honma, K. Nishimori, T. Seki, and M. Mizoguchi, "Short range MIMO communication," Proc. $3^{\text {rd }}$ European Conference on Antennas and Propagation, Berlin, Germany, pp. 1763-1767, Mar. 2009.

[2] K. Nishimori, N. Honma, T. Seki, and K. Hiraga, "On the transmission method for short-range MIMO communication," IEEE Trans. Vehicular Technol., vol. 60, no. 3, pp. 1247-1251, Mar. 2011. DOI:10.1109/TVT.2011.2113196 
[3] J.-S. Jiang and M. A. Ingram, "Spherical-wave model for short-range MIMO," IEEE Trans. Commun., vol. 53, no. 9, pp. 1534-1541, Sep. 2005. DOI:10. 1109/TCOMM.2005.852842

[4] K. Sakamoto, K. Hiraga, T. Seki, T. Nakagawa, and K. Uehara, "Performance evaluation of short-range MIMO using a method for controlling phase difference between each propagation channel," IEICE Trans. Commun., vol. E96-B, no. 10, pp. 2513-2520, Oct. 2013. DOI:10.1587/transcom.E96. B.2513

[5] K. Murata, N. Honma, K. Nishimori, and H. Morishita, "Analog eigenmode transmission for short-range MIMO,” IEEE Trans. Vehicular Technol., in press. DOI:10.1109/TVT.2015.2397194

[6] K. Murata, N. Honma, K. Nishimori, and H. Morishita, "Analog eigenmode transmission for $2 \times 2$ short-range MIMO,” Proc. IEEE AP-S International Symposium, Memphis, USA, pp. 476-477, Jul. 2014. DOI:10.1109/APS. 2014.6904569

[7] D. M. Pozar, Microwave Engineering, 4th ed., Wiley, New York, 2012.

[8] G. R. Simpson, “A generalized n-port cascade connection,” Proc. IEEE MTT-S International Microwave Symposium Digest, Los Angeles, USA, pp. 507-509, Jun. 1981. DOI:10.1109/MWSYM.1981.1129978

[9] K. Murata, N. Honma, K. Nishimori, and H. Morishita, "Bidirectional matching technique for Tx and Rx ports in short-range MIMO," 2014 AsiaPacific Microwave Conference (APMC), Sendai, Japan, pp. 498-500, Nov. 2014.

[10] http://www.mentor.com/pcb/hyperlynx/3d-em/.

[11] D. M. Pozar, "Microstrip antennas," Proc. IEEE, vol. 80, no. 1, pp. 79-91, Jan. 1992. DOI:10.1109/5.119568

[12] http://www.ieee802.org/11/.

\section{Introduction}

Short-range MIMO, an emerging utilization form of MIMO technology, is a promising avenue to realize a high-speed large-capacity short-range wireless communication by employing multiple antennas $[1,2,3]$. Currently, the simplification of SR-MIMO hardware-software configuration is being pursued for its practical usages, and fixed-weight analog decoding methods were developed utilizing the uniquely-determined channel property [4]. However, the conventional methods suffer from the two unavoidable problems, "capacity deterioration caused by array displacement" and "being valid only at optimal array configuration," that exclusively confine its applicable scope. In order to solve the problems, an analog eigenmode transmission technique using 180-degree hybrid couplers was proposed. It was theoretically proven that this technique could orthogonalize MIMO channels regardless of antenna spacing and Tx-Rx distance [5].

In this letter, its array parameter independency is quantitatively evaluated by experiment [6]. In addition, its frequency characteristics are newly reported. Consequently, it has been experimentally demonstrated that the proposed analogbased E-SDM (AE-SDM) can achieve more than 90-\% performance of ideal digital-based E-SDM (DE-SDM) regardless of the above-mentioned parameters. 


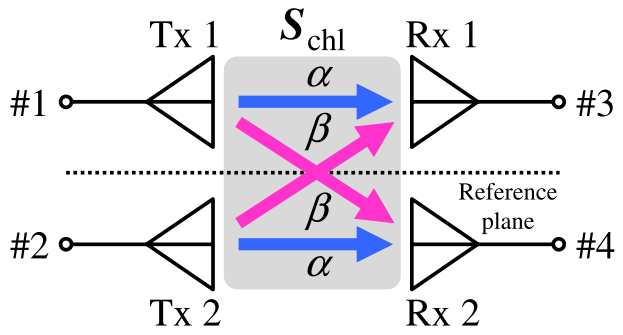

(a) Without hybrid.

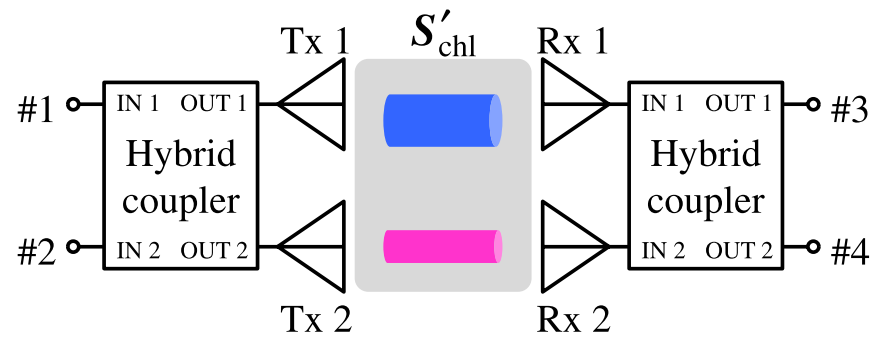

(b) With hybrids.

Fig. 1. Conceptual sketch of the proposed technique.

\section{Theory}

The theory of the proposed technique is briefly explained by taking a $2 \times 2$ SR-MIMO system for example. As shown in Fig. 1(a), if a SR-MIMO array is configured symmetrically with respect to the reference plane, a channel matrix, $\boldsymbol{S}_{\mathrm{chl}}$, becomes symmetrical; the channels between facing ports, $S_{31}$ and $S_{42}$, are equivalent as well as those between diagonal ports, $S_{41}$ and $S_{32}$. Therefore, by replacing them with constants, $\alpha$ and $\beta$, respectively, the channel matrix is expressed as

$$
\boldsymbol{S}_{\mathrm{chl}}=\left[\begin{array}{ll}
S_{31} & S_{32} \\
S_{41} & S_{42}
\end{array}\right]=\left[\begin{array}{ll}
\alpha & \beta \\
\beta & \alpha
\end{array}\right] \text {. }
$$

Next, hybrids are cascade-connected to both Tx/Rx sides as shown in Fig. 1(b). The transmission matrix of an ideal hybrid, $\boldsymbol{S}_{\mathrm{hyb}}$, is expressed as

$$
\boldsymbol{S}_{\text {hyb }}=\frac{j}{\sqrt{2}}\left[\begin{array}{cc}
1 & 1 \\
1 & -1
\end{array}\right]
$$

and an ideal hybrid has neither reflection at each port nor couplings between inputs and between outputs [7]. The complete channel matrix of the whole SR-MIMO system including the hybrids, $\boldsymbol{S}_{\text {chl }}^{\prime}$, is expressed as the product of the transmission matrices of the Tx/Rx hybrids and the channel matrix of the initial SR-MIMO system [8],

$$
\boldsymbol{S}_{\mathrm{chl}}^{\prime}=\boldsymbol{S}_{\mathrm{hyb}} \boldsymbol{S}_{\mathrm{chl}} \boldsymbol{S}_{\mathrm{hyb}}=-\left[\begin{array}{cc}
\alpha+\beta & 0 \\
0 & \alpha-\beta
\end{array}\right] .
$$

Equation (3) indicates that two parallel streams are formed between the pairs of the corresponding Tx/Rx ports. And, on the condition that the symmetry of the array configuration is maintained, array parameters such as the type of antenna, antenna spacing and Tx-Rx distance have no influence on the channel orthogonality. 
Although reflection and coupling components at both $\mathrm{Tx} / \mathrm{Rx}$ arrays are omitted in the derivation process of Eq. (3) due to space limitations, it was proven in our previous work that these factors had no impact on the channel orthogonalization by the proposed technique [9].

\section{Experiment}

\subsection{Experimental setup and condition}

Fig. 2(a) shows the SR-MIMO system configuration used for an experiment. The $\mathrm{Tx} / \mathrm{Rx}$ arrays consisted of two microstrip antennas (MSAs) shown in Fig. 2(b). The MSA was fabricated on a substrate with thickness of $1.56 \mathrm{~mm}$ and relative permittivity of 2.2. The size of the MSA and its feeding point were optimized so it was fully matched at the center frequency of $2.5 \mathrm{GHz}$. Just for reference, simulated half-value angles of E- and H-plane far-field radiation patterns of the MSA are 84.5 and 88.3 degrees, respectively. Regarding array parameters, antenna spacing, $d$, and Tx-Rx distance, $D$, were defined as the distances between feeding points of adjacent elements and between $\mathrm{Tx} / \mathrm{Rx}$ array substrates, respectively. As a hybrid used in the proposed technique, a rat-race coupler fabricated on the same substrate as the MSA was employed (Fig. 2(c)). In the figure, $\lambda_{\text {eff }}(87.8 \mathrm{~mm})$ is the effective wavelength at the center frequency calculated with the substrate parameters considered. The width of the ring was determined for a characteristic impedance of $\sqrt{2} Z_{0}\left(Z_{0}\right.$ is $50 \Omega$.) In the proposed SR-MIMO system, two rat-races were connected to the $\mathrm{Tx} / \mathrm{Rx}$ arrays and a measuring instrument via flexible cables from the back sides of the rat-races.

In the experiment, a submatrix representing transmission from $\mathrm{Tx}$ to $\mathrm{Rx}$ arrays in the S-parameter of the SR-MIMO system was defined as a channel matrix, and two patterns of channel matrices were measured. First, as the proposed AE-SDM, channels were obtained when the rat-races were connected. Second, channels were extracted when the $\mathrm{Tx} / \mathrm{Rx}$ arrays were directly connected to the measuring instrument, and after that, DE-SDM was implemented through post processing. Finally, channel capacities of AE-SDM and DE-SDM were calculated based on Shannon's theorem regarding a signal from a non-corresponding Tx port as interference [5]. In calculating capacities, the ratio of transmission power to receiving noise level was set to $40 \mathrm{~dB}$.

For reference, numerical simulations were also carried out to verify experimental results. For electromagnetic analysis, IE3D was used [10]. In the simulation, the SR-MIMO array identical to that of experiment was designed, and a channel matrix was extracted in the same manner as experiment. Regarding a hybrid part, an ideal (but frequency-dependent) rat-race consisting of distributedconstant lines was assumed separately from the array configuration. A channel matrix when hybrids cascade-connected was numerically analyzed based on the Sparameter theory [8]. Therefore, the structural influence of the hybrids were excluded from channel characteristics. 


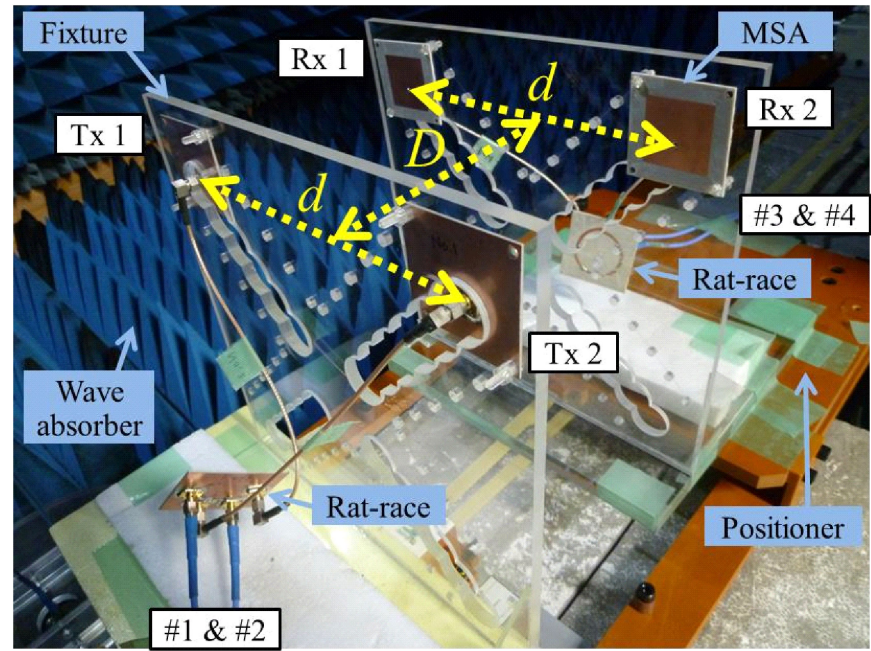

(a) System configuration.

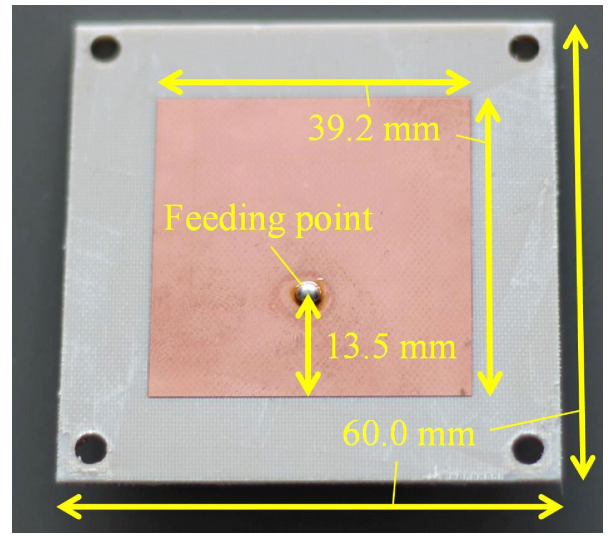

(b) Manufactured MSA.

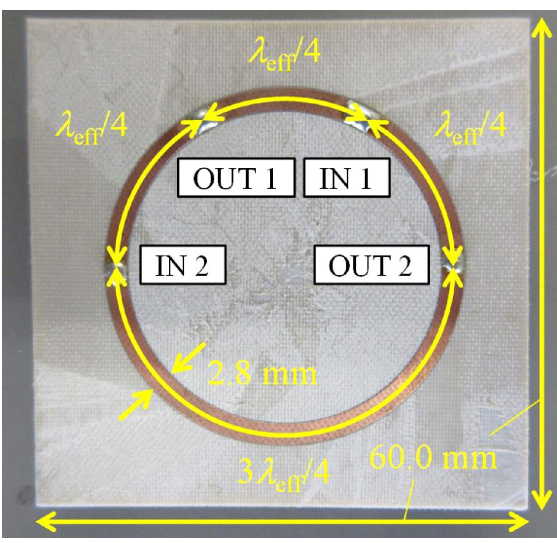

(c) Manufactured rat-race.

Fig. 2. Experimental setup.

\subsection{Experimental results}

\subsubsection{On array parameter independency}

Fig. 3(a) shows channel capacity versus antenna spacing. In the experiment, $d$ was varied to $81,112,143,174$ and $205 \mathrm{~mm}$, and $D$ was fixed to $120 \mathrm{~mm}$. In the figure, markers of circles, squares and triangles represent the channel capacities of AESDM, E-SDM and without any beamforming (shown as w/o BF), respectively. Also, simulation results are shown with different types of lines.

First, see the simulation results, and it is particularly notable that AE-SDM has exactly same properties with DE-SDM. This proves that AE-SDM allows a perfect two-stream parallel transmission in theory. Then, focusing on experimental results, it is confirmed that a channel capacity is dramatically improved by AE-SDM compared to $\mathrm{w} / \mathrm{o} \mathrm{BF}$ since interference channel components are suppressed by using rat-races as expressed as Eq. (3). As a result, channel capacity increases 2.4and 3.7-fold on average and at maximum when $d=81 \mathrm{~mm}$, respectively. Incidentally, it is observed that the deviation between experimental and numerical results of w/o BF becomes significant as $d$ increases. The cause of it is considered as follows; when $d$ is small, the hybrids are blocked by MSAs, therefore, they have less impact on channel characteristics. On the other hand, when $d$ is large, the hybrids are 
exposed and they generate more scattered waves. Especially, it appears that the ratraces work to suppress interference components when $d=205 \mathrm{~mm}$, that leads to such improvement in channel capacity. Likewise, it is thought that the slight difference between increase-decrease tendencies of experimental and simulation results of DE-SDM comes from such scattering effect. In comparison with DESDM, although AE-SDM should theoretically agree with DE-SDM, the capacity is deteriorated as the whole. The possible causes are cited as follows; (i) the impurity of channel orthogonality by manufacturing error of rat-races and array displacement, and (ii) mismatching and insertion losses by rat-races, cables and connectors. However, the capacity deterioration is retained only $8.4 \%$ on average over the analysis points of antenna spacing.

Fig. 3(b) shows channel capacity versus Tx-Rx distance. In the experiment, $d$ was fixed to $81 \mathrm{~mm}$ and $D$ was automatically changed from 60 to $180 \mathrm{~mm}$ at $10-\mathrm{mm}$ intervals using a positioner. Comparing AE-SDM with w/o BF, the significant capacity improvement, a 3.6-fold increase on average, can be seen as the previous result shows. Moreover, the capacity deterioration is retained $7.9 \%$ on average over the analysis range of Tx-Rx distance. These results successfully demonstrate that $\mathrm{E}$ SDM can be realized independently of both antenna spacing and Tx-Rx distance by the proposed technique.

\subsubsection{On wideband characteristics}

Fig. 3(c) shows channel capacity versus normalized frequency. In the experiment, the analysis frequency range was $\pm 2.5 \%$ from the center frequency, comparable to a possible bandwidth of a general MSA [11]. As for array parameters, $d$ and $D$ were fixed to 81 and $120 \mathrm{~mm}$, respectively. In capacity analysis, it was assumed that ideal DE-SDM could be implemented at every analysis frequency point while a frequency response of the rat-races was taken into account for AE-SDM. In the figure, the most interesting point is that AE-SDM keeps the capacities nearly equal to those of DE-SDM against frequency deviation in the simulation even though the frequency dependency of the rat-races was considered. Similarly, although the capacity deterioration is confirmed in the experiment because of the aforementioned causes, the deterioration rates are almost constant $(8.1 \sim 10.5 \%)$ over the analysis range. This indicates that the frequency response of the rat-races has a negligible effect on the capacity deteriorations. These results attribute to the wideband characteristics of a rat-race (its bandwidth is generally 20 30\% [7]), and as a consequence, the proposed technique can keep the channel orthogonalization effect over the broad band of frequencies, i.e. AE-SDM performance is only limited by antenna frequency dependency.

Fig. 3(d) shows a channel capacity with 5-\% bandwidth of each scheme. From the experimental results, it is found that AE-SDM can achieve a 3.3 times higher capacity than w/o BF, and moreover, a 91.7-\% performance of ideal DE-SDM. For supplementary information, according to [12], channel widths of wireless LAN (WLAN) related standards IEEE $802.11 \mathrm{n}$ and $11 \mathrm{ac}$ are at most approximately 1.7 and $0.8 \%$ (40 MHz at 2.4 and $5 \mathrm{GHz}$ bands, respectively) and $3.2 \%(160 \mathrm{MHz}$ at $5 \mathrm{GHz}$ band), respectively. These values can be sufficiently covered by the propose technique, therefore, its applicability has been suggested. 


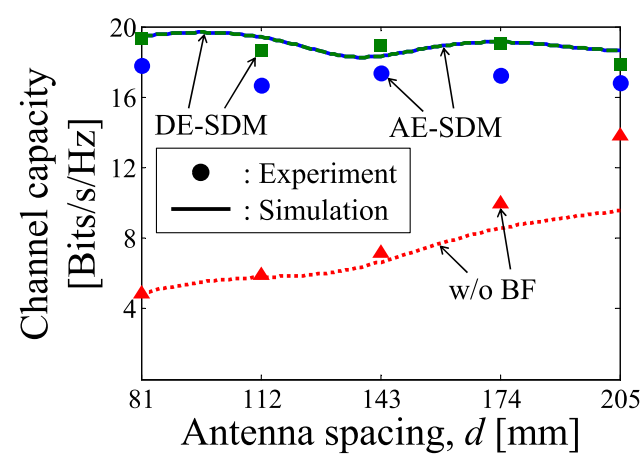

(a) Versus antenna spacing.

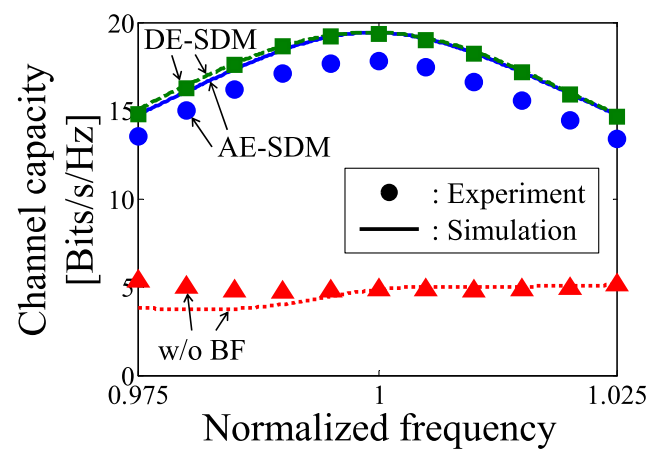

(c) Versus frequency.

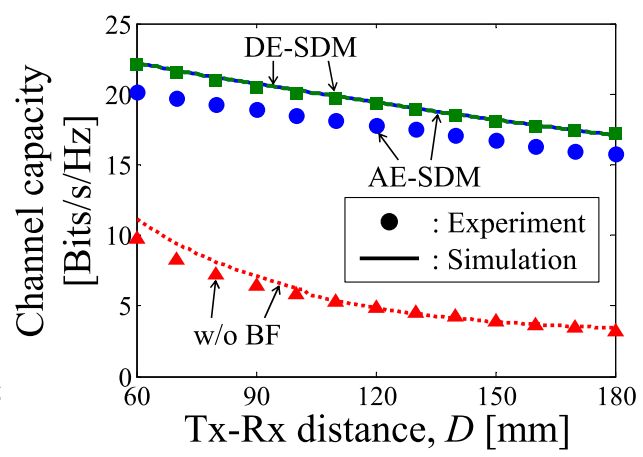

(b) Versus Tx-Rx distance.

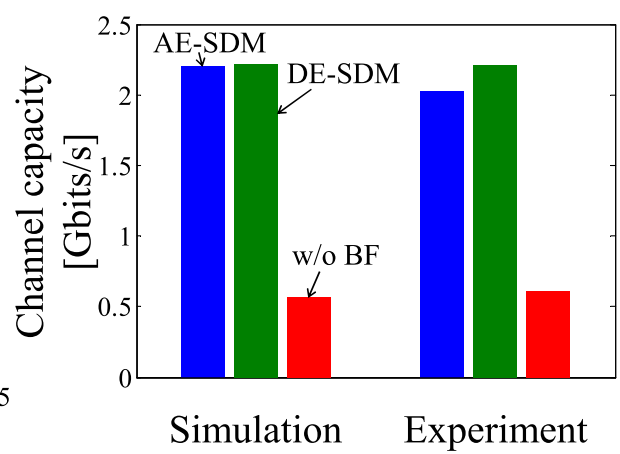

(d) With 5-\% bandwidth.

Fig. 3. Channel capacity characteristics.

\section{Conclusion}

The proposed technique can realize multi-stream parallel transmission using only passive analog components. Therefore, E-SDM can be performed by a fairly simple hardware configuration with neither digital beamformer nor active electrical requirements. In this letter, the two huge advantages of AE-SDM, array parameter independency and wideband characteristics, have been experimentally evaluated. On array independency, it has been demonstrated that AE-SDM achieves almost the same level of the capacity of DE-SDM with only 8.4- and 7.9-\% degradations on average over analysis ranges of antenna spacing and $\mathrm{Tx}-\mathrm{Rx}$ distance, respectively. Furthermore, it has been clarified that AE-SDM can achieve a 91.7-\% performance of ideal DE-SDM over the 5-\% relative bandwidth, that covers the channel widths of some existing WLAN standards. From these results, it has been confirmed that E-SDM can be successfully demonstrated by the proposed technique independently of the above parameters, and finally, the effectiveness of it has been experimentally verified.

\section{Acknowledgement}

This research was partially supported by JSPS KAKENHI (25709030). 